Both the humoral and cellular immune systems were affected by rituximab treatment. Levels of IgM, IgE, anti-dsDNA and antiC1q antibodies decreased upon treatment. Conversely, there was a considerable increase in the numbers of activated $T$ cells as well as regulatory $\mathrm{T}$ cells; the authors attributed this finding to symptomatic improvement, which resulted in decreased T-cell traffic to inflammatory sites. The authors call for future studies to determine the influence of cyclophosphamide on the immunological effects observed in their study.

Original article Vallerskog T et al. (2007) Treatment with rituximab affects both the cellular and the humoral arm of the immune system in patients with SLE. Clin Immunol 122: 62-74

\section{Elevated IFN- $\gamma$ can indirectly cause bone loss in osteoporosis}

Activation of the immune system in some pathological conditions leads to increased secretion of factors that influence bone turnover. For example, activated $\mathrm{T}$ helper type 1 lymphocytes secrete interferon (IFN)- $\gamma$, which has potent effects on osteoclastogenesis in vivo and in vitro. IFN- $\gamma$, however, is known to cause both bone resorption and bone formation, and studies that examined its effects on bone have produced contradictory results.

Gao and colleagues have shown that the net effect of IFN- $\gamma$ on bone depends on the balance between two opposing actions: IFN- $\gamma$ blocks osteoclastogenesis (and hence encourages bone formation) via a direct action on osteoclast precursors, but simultaneously stimulates osteoclastogenesis (and hence bone resorption) via an indirect pathway-IFN- $\gamma$ stimulates antigen-dependent T-cell activation, which results in increased T-cell secretion of the osteoclastogenic factors RANKL (receptor activator of NF-kB ligand) and tumor necrosis factor.

The authors analyzed the net effect of IFN- $\gamma$ in three mouse models of pathological bone loss-lipopolysaccharide injection (which mimics bacterial infection), inflammation caused by silencing of transforming growth factor- $\beta$ signaling in T cells, and ovariectomy (which causes estrogen deficiency), respectively. The authors found that in all three models the indirect pro-osteoclastogenic and boneresorptive effects of IFN- $\gamma$ prevail over the direct anti-osteoclastogenic activity.
The authors suggest that inhibition of IFN- $\gamma$ signaling could provide a novel approach for the concomitant prevention of inflammation and bone loss in common osteoporotic conditions, including estrogen deficiency, infection and inflammation.

Original article Gao Y et al. (2007) IFN- $\gamma$ stimulates osteoclast formation and bone loss in vivo via antigen-driven T cell activation. J Clin Invest 117: 112-132

\section{Intra-articular hyaluronan derivative is a potential treatment for hip OA}

An open-label, pilot study has demonstrated that intra-articular injections of hylan G-F 20-a cross-linked, high-molecular-weight derivative of hyaluronan-could potentially be an effective treatment for hip osteoarthritis (OA). This study is notable because the clinical efficacy of intra-articular injections of hyaluronan and its derivatives is a contentious issue.

In total, 56 patients with hip OA received either one or two intra-articular injections of hylan G-F 20. Second injections were administered to 31 of the 56 patients (1,2 or 3 months after the first injection) because the first injection was ineffective or their symptoms recurred. Concomitant treatment with NSAIDs, corticosteroids, aspirin above $325 \mathrm{mg} /$ day and analgesics other than paracetamol were prohibited. Oral disease-modifying drugs for $O A$ were permitted if the dose had been stable for $\geq 3$ months before the study.

At 90 days after the first injection, 30 patients met the Outcome Measures in RheumatologyOsteoarthritis Research Society Initiative standardized criteria for response to treatment, 20 patients achieved the 'patient-acceptable symptom state', and 25 patients had pain reduction greater than the 'minimal clinically important improvement'. At 90 days after the most recent injection, 29 patients had a decrease in walking pain.

Conrozier and colleagues caution that the lack of a control group in their trial means that the efficacy of intra-articular hylan G-F 20 remains unproven. A controlled, double-blind trial of intra-articular hylan GF-20 is currently underway.

\footnotetext{
Original article Conrozier T et al. (2006) Clinical response to intra-articular injections of hylan G-F 20 in symptomatic hip osteoarthritis: the OMERACT-OARSI criteria applied to the results of a pilot study. Joint Bone Spine 73: 705-709
} 